\title{
BMJ Open Does early intervention improve outcomes in physiotherapy management of lumbar radicular syndrome? A mixed-methods study protocol
}

\author{
Michael Reddington, ${ }^{1}$ Stephen J Walters, ${ }^{2}$ Judith Cohen, ${ }^{3}$ Susan Baxter ${ }^{4}$
}

To cite: Reddington $\mathrm{M}$, Walters SJ, Cohen J, et al. Does early intervention improve outcomes in physiotherapy management of lumbar radicular syndrome? A mixed-methods study protocol. BMJ Open 2017;7:e014422.

doi:10.1136/bmjopen-2016014422

- Prepublication history for this paper is available online. To view these files please visit the journal online (http://dx.doi.org/10.1136/ bmjopen-2016-014422).

Received 22 September 2016 Accepted 26 September 2016

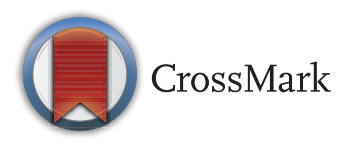

${ }^{1}$ Therapy Services Outpatient Department, Northern General Hospital, Sheffield, UK

${ }^{2}$ Designs, Trials and Statistics ScHARR, University of Sheffield, Sheffield, UK ${ }^{3}$ Clinical Trials Research Unit ScHARR, University of Sheffield, Sheffield, UK ${ }^{4}$ Section of Public Health ScHARR, University of Sheffield, Sheffield, UK

Correspondence to Michael Reddington; MReddington1@sheffield. ac.uk

\begin{abstract}
Introduction: Lumbar radicular syndrome (LRS) can be a painful and debilitating condition. The optimum management strategies and their timing remain elusive despite extensive research. Surgery provides good short-term outcomes but has concomitant risks and costs. Physiotherapy is commonly practised for patients with LRS but its effects remain equivocal and there is a lack of consensus on the type, duration and timing of physiotherapy intervention. There is a lack of high-quality evidence into new and innovative management strategies and the timings of those strategies for LRS. This pilot trial is an essential preliminary to a definitive randomised controlled trial (RCT) assessing the effectiveness and costeffectiveness of early physiotherapy intervention for patients with LRS. The study will test the protocol, the intervention, the use of outcome measures and the ability to set-up and run the trial to enable refinement of a future definitive RCT.
\end{abstract}

Methods and analysis: This is a mixed-methods study encompassing an external pilot RCT with integrated qualitative interviews with patients, clinicians and other key stakeholders. 80 patients will be recruited from primary care and randomised, after consent into 1 of 2 groups. Both groups will receive individually tailored, goal orientated physiotherapy. The usual care group will begin their physiotherapy 6 weeks after randomisation and the intervention group at 2 weeks after randomisation. Outcome measures will primarily be feasibility parameters including the ability to recruit and retain patients and to deliver the intervention. Data will be collected at baseline, and 6 , 12 and 26 weeks following randomisation.

Ethics and dissemination: The study has received favourable ethical review from the East of Scotland Research Ethics Service (EOSRES) on the 20 August 2015 (15/ES/0130). Recruitment began on the 1 March 2016 and is expected to close in January 2017. Data collection is anticipated to be complete in July 2017. The study results will be made available to participants, clinicians involved in the study and the wider clinical community through publication in a peer reviewed journal and at conference presentations.

Trial registration number: ISRCTN: 25018352 , Pre-results; Clinical Trials.Gov: NCT02618278 Document version V1.1 23.9.2016.

\section{Strengths and limitations of this study}

- This feasibility study will inform the design and conduct of a definitive randomised controlled trial of an early physiotherapy intervention for the treatment of lumbar radicular syndrome (LRS).

- The intervention is a safer, cheaper alternative to surgery for patients with LRS and should be easy to adopt and deliver within current treatment pathways.

- The qualitative component of the study will enable patient and stakeholder views to be taken into consideration and they will be used to inform modifications to the intervention and design of the definitive trial.

- Recruitment in the feasibility study will be from 14 General Practitioner (G.P) practices in the north of England with diverse demographic populations. Generalisability of the results will need to be considered when planning a larger trial including other areas of the UK.

\section{INTRODUCTION}

Lumbar radicular syndrome (LRS) is a painful and often disabling condition, which is usually of benign causation. It can be selflimiting and may last a short time with no significant sequelae or, in some individuals it can be severely painful and disabling in the long term. The reasons for the wide variations in presentation, outcome and duration in patients are not fully understood. ${ }^{1}$

LRS is perhaps better known as sciatica, although sciatica does not encompass neural dysfunction from the upper lumbar nerve roots and thus, the term LRS is preferred. LRS is defined as leg pain in an area served by one or more spinal nerve roots and can be accompanied by neurological deficit such as paraesthesia, anaesthesia and myotomal weakness. ${ }^{2}$ There are many and varied estimates of prevalence of LRS ranging from $1 \%$ to $43 \%{ }^{3}$ 
LRS is a major cause of disability, work loss and presentation to healthcare. ${ }^{4}$ The Health Council of the Netherlands estimated a cost of 2.9 billion Guilders to the Dutch economy in 1999 (around $€ 1.5$ billion at September 2015 exchange rate). The costs of surgery for LRS (microdiscectomy and laminectomy) were found to add $\$ 5$ billion to the overall cost of back pain and LRS in the USA in $2004 .{ }^{5}$ The cost of physiotherapy for a group of patients who went on to have surgery for LRS accounted for $11 \%$ of the total preoperative costs or on average $\$ 379$ per patient. The mean cost of imaging alone accounted for $31 \%$ of costs or $\$ 1067$ for each patient, ${ }^{6}$ illustrating the relatively small costs of physiotherapy compared with other, non-therapeutic costs.

The management of LRS is a much debated and contentious area. Surgery for patients with LRS has been advocated, with good reported outcome. ${ }^{7}$ However, significant number of patients never have any substantial relief from surgery, ${ }^{8}{ }^{9}$ with unsatisfactory outcomes in over $20 \%$ of patients at 5 years, irrespective of the type of treatment they receive, including surgery. ${ }^{10}$ Although, it is not known with any certainty how long to wait to allow spontaneous resorption of the inter vertebral disc (IVD) prolapse, it has been suggested that by 12 weeks $75 \%$ of LRS sufferers will have symptomatically resolved. ${ }^{11}$ The favourable effects of conservative treatment for LRS have been demonstrated with $90 \%$ of patients improving within 12 weeks after onset. ${ }^{12} \mathrm{~A}$ "wait and see' approach is often advocated but a significant number of people suffering with LRS do not recover in the short or medium term and the optimal window for surgery can then be missed. Lewis et $a l^{13}$ note the importance of early treatment in order to prevent chronic symptom development and the ensuing resistance to treatment and costs.

While physiotherapy for LRS has been advocated, there is a lack of consensus on the type, duration or timing of intervention. ${ }^{14}$ There are numerous physiotherapy approaches to low back pain (LBP) and LRS, none of which have significant degree of scientific rigour surrounding them.

It is known that patients prefer and have improved outcomes with early intervention physiotherapy for LBP. $^{15-17}$ Delayed initiation of physiotherapy for patients with LBP in primary care is associated with increased cost and increased healthcare consumption. ${ }^{18}$ These findings were echoed by Gellhorn ${ }^{19}$ who found that patients who received physiotherapy $<4$ weeks after onset of their LBP had lower healthcare usage (and associated costs) than those who received physiotherapy more than 3 months after onset. Although such evidence does not directly exist for the provision of physiotherapy for patients with LRS, it may be suggested that early intervention is preferable.

\section{Aims and objectives}

The overall aim for the study is to investigate the feasibility of undertaking a fully powered, multicentre randomised controlled trial (RCT) to determine the effectiveness and cost-effectiveness of early intervention physiotherapy for patients with LRS.

\section{Objectives}

The objectives of the pilot trial fall into two categories. First, process objectives will allow the analysis of the practical and logistical aspects of setting up and running the study. Second, the feasibility objectives will provide data on recruitment, the use of outcome measures, randomisation and data collection and the delivery of the training and intervention. This information will be used to inform the definitive RCT.

\section{Process objectives}

1. To test the feasibility, practicality, safety and acceptability of the study design and protocol.

2. Demonstrate the ability to set-up and recruit in primary care centres.

3. To assess the feasibility of delivering the early intervention within the time parameters ( 2 weeks for the intervention group, 6 weeks for the usual care group).

4. Demonstrate a recruitment rate of seven patients per month in a maximum of 14 G.P centres, equal to a rate of 0.5 of a participant per centre, per month.

5. Demonstrate the ability to organise $75 \%$ of physiotherapy appointments within 2 weeks of randomisation.

6. Patient attendance at $66 \%$ of individual sessions.

7. $75 \%$ of patients randomised to early intervention have their first session within 20 days of randomisation.

8. Patient attrition rate of $<25 \%$ over the course of the study.

9. Outcome measurement return rate of $80 \%$ at $6 / 52$ follow-up.

\section{Feasibility objectives}

1. To determine the acceptability of the intervention to patients and clinicians.

2. Demonstrate acceptability of the primary and secondary outcome measures to patients and clinicians.

3. To inform the sample size calculation for the definitive trial.

\section{METHODS}

Design

Mixed-methods study comprising an external pilot RCT augmented by semistructured interviews with key stakeholders.

\section{Quantitative}

Recruitment will occur in three, 20-week cycles, illustrated in table 1 . In each of the three cycles there will be 12 weeks of active recruitment followed by up to 8 weeks for treatment to be completed. A 2-week period between cycles will provide time to reflect and analyse 


\begin{tabular}{lll} 
Table 1 & Recruitment cycles & \\
\hline Cycle A & & \\
\hline Week & Intervention & Usual care \\
\hline 1 & Recruitment and treatment & Recruitment \\
2 & & \\
3 & & \\
4 & & \\
5 & & Recruitment and \\
6 & & \\
7 & & \\
8 & & \\
9 & & \\
10 & & \\
11 & & \\
12 & & \\
13 & Treatment \\
14 & & \\
15 & & \\
16 & & \\
17 & & \\
18 & & \\
19 & & \\
20 & & \\
21 & Analyse and refine & \\
Cycle B & & \\
\hline
\end{tabular}

on the results from the stakeholder interviews and other feedback.

\section{Qualitative}

In-depth, semistructured interviews will be carried out with consenting patients in the intervention and control arms of the study. This will involve interviewing the patients before and after the intervention delivery. The treating physiotherapists will be interviewed before they begin 3 days of intervention training and also at the completion of the study. Other stakeholders in the process of delivering the intervention will also be asked for their feedback during the course of the study either through interviews or in the case of G.Ps, a weekly email forum. The aims of the qualitative elements of the study are first to compare and contrast the experiences of patients in the intervention and control groups of the pilot trial, and second to obtain the views and experiences of patients with regards to the study processes. In particular, the design, methods, randomisation and intervention.

A total of 80 patients with LRS will be recruited and each individual will be involved in the study for 6 months. A flow chart illustrating the study is found in figure 1.

\section{Ethical review and trial registration}

The study is being conducted in accordance with the Declaration of Helsinki and local governance requirements. The trial has been registered with current controlled trials ISCTRN number: 25018352 and Clinical Trials.Gov number NCT02618278.

\section{Setting}

Recruitment into the study will take place in a primary care setting with 14 G.P practices having committed to act as recruitment centres. The physiotherapy intervention will take place at one existing primary care treatment site.

\section{Participants (inclusion and exclusion criteria)}

Patients will be eligible to participate in the study if they are over 18 years of age and have unilateral LRS. Patients will be ineligible to participate if they have any existing condition which will affect their ability to undergo rehabilitation, for instance previous Cerebrovascular accident (CVA) with physical and/or psychological sequelae which would prevent rehabilitation, proven vascular claudication of lower limbs which could mimic LRS, spinal fracture, ongoing cancer and inadequate English language skills preventing questionnaire and interview completion.

\section{Sample size}

It has been recommend that an external pilot study has at least 70 measured subjects (35 per group) when estimating the SD for a continuous outcome. ${ }^{20}$ A sample size of 80 patients, with $\sim 10 \%$ allowance for lost to follow-up allows the SD of an outcome to be estimated to within a precision of $\sim \pm 16 \%$ of its true underlying value with $95 \% \mathrm{CI}$.

\section{Qualitative sampling}

Although it is difficult to judge how many participants will be required for interview until data saturation is reached, it is estimated that around 10-15 interviewees will be required per study arm. Ritchie ${ }^{21}$ outlines several factors that can influence decision making regarding the size of samples in qualitative research. Two key factors are the heterogeneity of the study population and the available resources. It is expected that in this study population there should be a degree of homogeneity, in that all of the participants are suffering with LRS. Individual characteristics such as sex, age and duration of onset will be considered in order to achieve diversity in the sample.

\section{Recruitment and consent}

Potential participants will be given a patient information sheet when they see their G.P for help with their LRS. A brief screening process will take place when the potential participant contacts the research team and consent will obtained during a face-to-face meeting, before baseline measures are collected.

\section{Randomisation}

Information from the baseline data set will be used to randomise the participant. Randomisation will be 


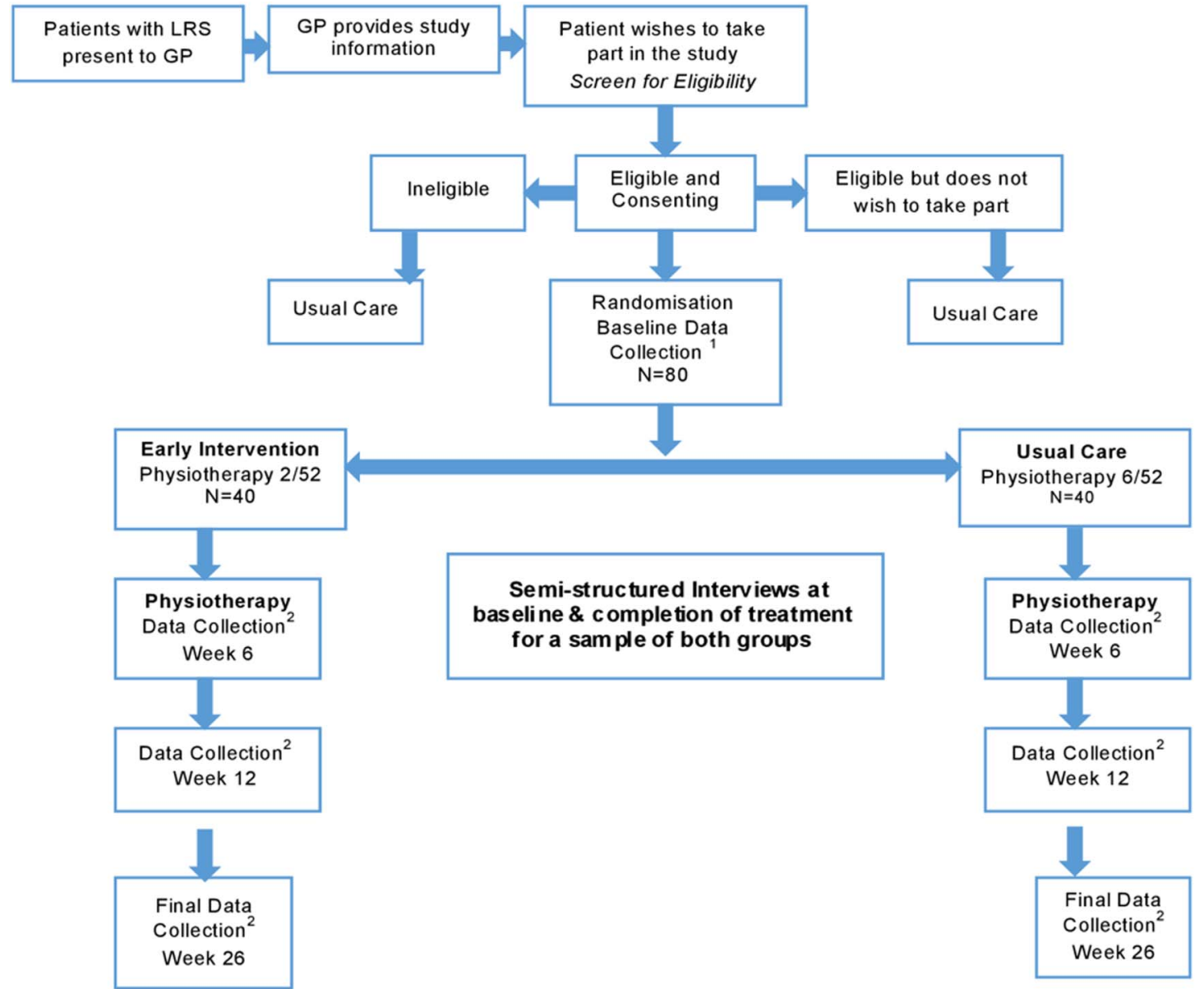

Data collection to include: Baseline demographics, Medical history, ODI, VAS, STB, EQ5D-5L Data Collection to include ODI, VAS, EQ5D-5L

Figure 1 Flow chart illustrating the recruitment and randomisation process. GP, general practice; LRS, lumbar radicular syndrome; ODI, Oswestry Disability Index; STB, STarT Back screening tool.

achieved by a web-based randomisation system with one stratification factor (Oswestry Disability Index (ODI)) with three levels based on ODI severity; 'mild \& moderate' $(\leq 22-40 \%)$, 'severe' $(>40-60 \%)$ and 'crippled' $(>60-80 \%)$. A blinded block size will be used to minimise predictability. The participants will be informed, within one working day of their consent and randomisation of their group allocation. With a study of a complex intervention such as the one described, it is very difficult to blind either patients or clinicians to the treatment allocation, as it is obvious to clinician and patient that they are receiving an intervention at either 2 weeks or 6 weeks. In an effort to minimise bias, both groups of patients will receive treatment based on the same assessment and treatment framework.

\section{The intervention}

The goal-orientated physiotherapy regimen for both groups will be tailored to the individuals' requirements. Participants will be assessed using a biopsychosocial approach based on seven different elements; neurological dysfunction, ${ }^{22}$ motor control of movement of the lumbar spine and pelvis, ${ }^{23}{ }^{24}$ movement restriction in the lumbar spine and pelvis, ${ }^{25}$ psychological barriers to recovery ${ }^{26-28}$ advice and education ${ }^{29}{ }^{30}$ functional-based exercise $^{31}$ and pain. ${ }^{32}$ Participants will receive up to six sessions of physiotherapy over an 8-week period or until they have achieved their predetermined goals.

The seven elements of assessment and treatment are neither exclusive nor exhaustive but reflect the complexity of the clinical reality of a patient presenting with LRS. The Medical Research Council (MRC) guidance for developing and evaluating complex interventions has, therefore, been adopted as the principal framework around which the study has been based. $^{33}$

Implementation fidelity testing will be carried out in order to assess the treating clinicians are delivering what is intended by the protocol. ${ }^{34}$ An independent assessor will review video footage of physiotherapist and participant session in order to assess implementation fidelity.

Patients are allowed to withdraw from treatment at any point and this will have no detrimental effects on their ongoing healthcare. 
Table 2 Screening and outcome measurement collection

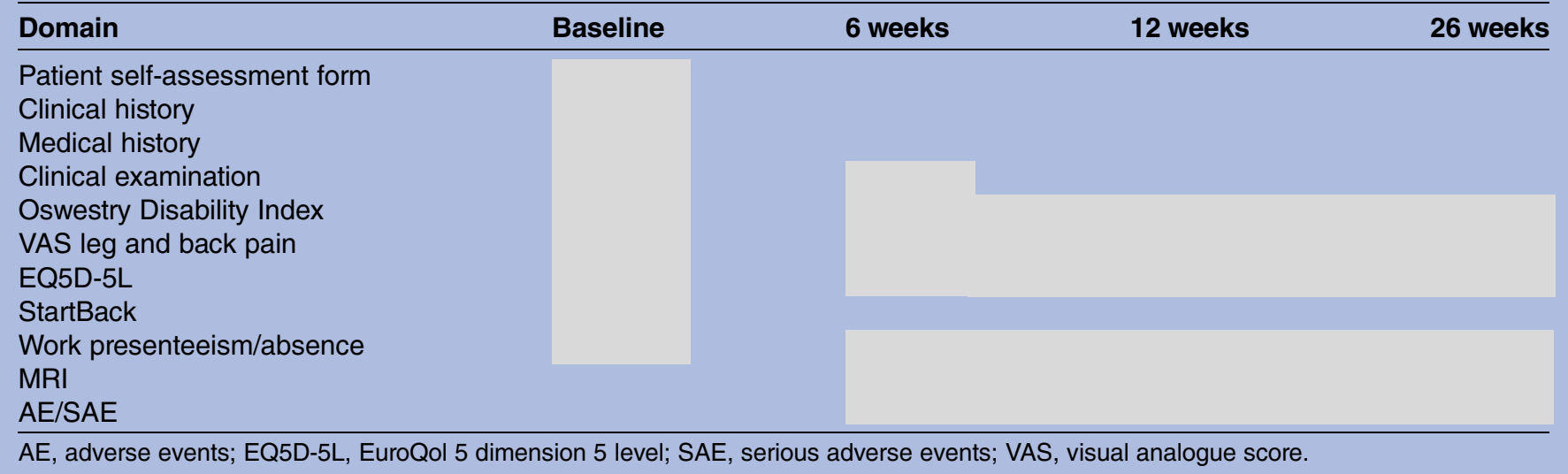

\section{Physiotherapist training and support}

Three physiotherapists will be recruited from the physiotherapy service provider. They will undergo 3-day (20 hours) training in each of the seven elements of the assessment and intervention to promote and facilitate self-management, function, pacing advice and optimum analgesic advice together with equipping the patient to cope with their symptoms optimally.

\section{Data collection}

Patients will be asked to complete baseline self-report and screening measures outlined in table 2 at the time of consent by the chief investigator or research nurse. These will include an initial self-assessment form including anthropometric data including gender, height, weight, socioeconomic status (work and any time off work due to the LRS). Participants will also be asked about their specific functional goals of undertaking physiotherapy. This baseline data will be used, in conjunction with a clinical assessment undertaken at their initial assessment, to formulate an individual treatment plan.

\section{Data management}

Patient identifiable data will be entered onto a bespoke, secure study database provided by the University of Sheffield (UoS) Clinical Trials Research Unit (CTRU), who adheres to Standard Operating Procedures (SOPs) relating to all aspects of data management including data protection and archiving. Patients will be given a unique study number on consenting to enter the study and they will be identified using their participant study number only. All hard copy data will be stored in a locked filing cabinet in accordance with data protection requirements for the retention of research data and UoS and Sheffield Teaching Hospitals NHS Foundation Trust (STHFT) data management policies. Data will not be shared with anyone outside of the direct research team (chief investigator, CTRU and supervisors). Access to data, including the trial master file, will be restricted to the sponsor.

\section{Primary and secondary outcome measures}

The primary outcome measures for the study will evaluate the feasibility of a range of factors. These will include the recruitment of patients into the study in a timely manner, in particular the ability to identify eligible patients and their willingness to be randomised. The recruitment rate and initiation of treatment within 2 weeks for intervention arm and 6 weeks for usual care arm will be noted. Ensuring patient safety and acceptability of the intervention to patients and clinicians and acceptability of the trial protocol. The practicalities of providing the physiotherapy and patient adherence to the intervention and in addition, the number of patients being referred into the secondary care system will be noted and costed accordingly. The number of patients undergoing surgery for their LRS will be recorded and costed as will the number of adverse events $(\mathrm{AE})$ and serious adverse events (SAE). Finally, the time taken from randomisation to physiotherapy treatment initiation will also be recorded.

\section{Data analysis plan}

As the trial is a pragmatic parallel group RCT, data will be reported and presented according to the CONSORT statement. ${ }^{35}$ The statistical analyses will be performed on an intention-to-treat basis. As a feasibility study, the main analysis will be mainly descriptive and will focus on CI estimation and not formal hypothesis testing. We will report rates of consent, recruitment and follow-up by centre and by randomised group. Outcome measures will be summarised overall and by randomised group. We will use the data from this feasibility study to estimate the consent rate, attrition rate, and the variability of the continuous outcomes (eg, leg and back pain VAS, ODI, EQ-5D) in the trial population and use this information to inform the sample size calculation for the definitive RCT. Since the intervention is therapist led we shall also use the data to estimate the intra-cluster correlation (ICC). We will also include, as part of the feasibility analysis, estimation of the effect size for the 12-week VAS-pain outcome (the probable primary end point for the definitive study) with CI estimates to check that the 
likely effect is within a clinically relevant range (as confirmation that it is worth progressing with the full trial). This information along with the acceptability of the study design and protocol to patients, therapists and G. Ps; the safety of the intervention; patient recruitment and consent/retention rates will enable us to determine whether or not the definitive RCT is feasible, within a satisfactory timescale and cost envelope using the UK centres alone. The time from randomisation to start of physiotherapy will be summarised by randomised group.

\section{Qualitative analysis}

The constant comparative method will be used for the analysis of the interview data. ${ }^{36}$ A preliminary thematic analysis $^{37}$ will be undertaken after three interviews by the lead author. This will take the form of category generation by defining units of data code generated by the interviews. Category generation will draw on both the interview data from the interviewee as well as the notes and reflections of the interviewer. The former aims to gain an insight of the meanings, views and opinions of the interviewee. The latter allows to allow the researcher to build on insights drawn from the interview in terms of the research area being addressed. ${ }^{38}$

Findings from interviews and feedback from participants and clinicians will be used after the 1st cycle of recruitment to inform changes to the intervention and processes for the 2nd cycle of recruitment. This will be repeated for the 2nd recruitment cycle to inform the 3rd cycle of recruitment. In this way it is envisaged that at the end of the 3rd and final cycle, the processes, intervention and design will be ready to form the basis of a full RCT. Changes will be made in discussion with the trial management group (TMG) and protocol amendments requiring ethical approval will be sought as appropriate. Subsequent interviews will be analysed by the researcher on an iterative basis, whereby, the ongoing analysis, along with the reflexive diary, will be used to identify new issues to explore with successive interviewees. Atlas.ti software will be used to manage the qualitative data.

\section{Mixed methods}

A mixed-methods approach has been adopted in order to gain an insight into the defined feasibility objectives and to add depth of understanding as to why aspects of the intervention or study process are working or not. In doing so it is hoped that more than a sum of the parts of the quantitative and qualitative components of the research alone will be realised.

Integration of the findings of the quantitative and qualitative components, gathered throughout the study will occur at the analysis stage. A mixed-methods matrix ${ }^{39}$ will be used for analysis of data from individual cases in terms of quantitative and qualitative findings.

\section{Health economic analysis}

An exploratory health economic analysis will be undertaken to inform a full-economic analysis for the future, full-scale trial. Patients will be asked to complete a short, self-report survey on costs that they have incurred as a direct consequence of their LRS. The factors on the analysis include time absent from work, analgesic requirements and cost, cost of any adjuncts used and costs incurred as a result of paying for care.

\section{Trial organisation and management}

The study is sponsored by STHFT in collaboration with the School of Health and Related Research (ScHARR), the UoS. The lead researcher (MR) will act as chief investigator with responsibility for project management in accordance with STHFT and UOS SOPs and will be overseen by the TMG.

A Trial Steering Committee (TSC) will be appointed and comprise of an independent chair, two patient representatives, a general practice (GP) representative, and clinicians with experience and expertise in the field of spinal disorders, including LRS. The TSC will review safety and progress of the trial and make recommendations to the TMG about study continuation.

A Data Monitoring and Ethics Committee (DMEC) is not required as physiotherapy is routine treatment for this patient group, the intervention changes the timing only and as such was considered low risk.

\section{Dissemination}

The results of the study will be presented initially to the patients who have taken part in the study and the participating clinicians. A report will be submitted to the funder of the study outlining the process and results. The results will be available via the study website at the host institute: http://www.sheffield.ac.uk/scharr/ sections/dts/ctru/polar. The results will be presented nationally/ internationally at conferences and through peer review, open access journals, subject to acceptance. Furthermore, the findings will be presented locally at musculoskeletal training events for physiotherapists and G.Ps. A key facts summary will be provided for the local musculoskeletal service commissioners.

\section{DISCUSSION}

There is a lack of high-quality evidence about the optimal way to treat patients with LRS. This study is a preliminary step towards establishing if a novel physiotherapy intervention, delivered within 2 weeks of referral from the G.P is an effective treatment for these patients. This study has been designed to assess the feasibility of a future, full-scale trial and will not investigate effectiveness of the intervention. The definitive RCT will be informed by the results of the study and will investigate the effectiveness and cost-effectiveness of the intervention, with the potential for patients to have better outcomes with avoidance of surgery and long-term problems.

Contributors MR instigated the idea for the study, developed the funding proposal and applied for funding. MR also developed the protocol, 
intervention handbook, ethical clearance and acts as chief investigator for the study. SJW is the primary PhD supervisor for MRs' fellowship and contributed to the study conception and design, and writing of the protocol. $\mathrm{JC}$ is an academic supervisor for MR and has provided specific guidance on protocol development, regulatory approvals and the design of the study. SB provided input regarding the qualitative and mixed-method design and analysis aspects of the study. All authors read and commented on drafts, and approved the final version of the manuscript.

Funding The lead author (MR) has received a personal Clinical Doctoral Research Fellowship award from the National Institute of Health Research (NIHR) and Health Education England (HEE), award number: CDRF-2014-05-046.

Disclaimer This paper presents independent research funded by the NIHR. The views expressed are those of the author(s) and not necessarily those of the NHS, the NIHR or the Department of Health.

Competing interests All authors have completed the Unified Competing Interest form at http://www.icmje.org/coi_disclosure.pdf (available on request from the corresponding author) and declare (1) No financial support for the submitted work from anyone other than their employer; (2) No financia relationships with commercial entities that might have an interest in the submitted work; (3) No spouses, partners or children with relationships with commercial entities that might have an interest in the submitted work; (4) No non-financial interests that may be relevant to the submitted work.

Ethics approval Ethical approval was received from NHS Scotland, East of Scotland Research Ethics Service in August 2015 (REC reference 15/ES/ 0130)

Provenance and peer review Not commissioned; peer reviewed for ethical and funding approval prior to submission.

Data sharing statement As the paper relates to a study protocol, there are no additional data sets available as yet.

Open Access This is an Open Access article distributed in accordance with the terms of the Creative Commons Attribution (CC BY 4.0) license, which permits others to distribute, remix, adapt and build upon this work, for commercial use, provided the original work is properly cited. See: http:// creativecommons.org/licenses/by/4.0/

\section{REFERENCES}

1. Suri P, Carlson MJ, Rainville J. Nonoperative treatment for lumbosacral radiculopathy: what factors predict treatment failure?. Clin Orthop Relat Res 2014;473:1931-9.

2. Koes $Æ$ Bart W, van Tulder MW PW. The diagnosis and treatment of sciatica. Br Med J 2007;334:1313-17.

3. Konstantinou K, Dunn KM. Sciatica review of epidemiological studies and prevalence estimates. Spine (Phila Pa 1976) 2008;33:2464-72.

4. Waddell G. The back pain revolution. 2nd edn. Edinburgh: Churchill Livingstone, 2004:1-7.

5. Deyo RA, Mirza SK, Martin BI, et al. Trends, major medical complications, and charges associated with surgery for lumbar spinal stenosis in older adults. JAMA 2010;303:1259-65.

6. Daffner SD, Hymanson HJ, Wang JC. Cost and use of conservative management of lumbar disc herniation before surgical discectomy. Spine J 2010;10:463-8.

7. Jacobs $\mathrm{WCH}$, van Tulder $\mathrm{M}$, Arts $\mathrm{M}$, et al. Surgery versus conservative management of sciatica due to a lumbar herniated disc: a systematic review. Eur Spine J 2011;20:513-22.

8. Haugen A, Grøvle L, Brox J, et al. Estimates of success in patients with sciatica due to lumbar disc herniation depend upon outcome measure. Eur Spine J 2011;20:1669-75.

9. Lurie JD, Tosteson TD, Tosteson ANA, et al. Surgical versus non-operative treatment for lumbar disc herniation: eight-year results for the Spine Patient Outcomes Research Trial (SPORT). Spine 2013;39:3-16.

10. Lequin $\mathrm{MB}$, Verbaan $\mathrm{D}$, Jacobs $\mathrm{WCH}$, et al. Surgery versus prolonged conservative treatment for sciatica: 5-year results of a randomised controlled trial. BMJ Open 2013;3:e002534.

11. Vroomen PC, de Krom MC, Knottnerus JA. Predicting the outcome of sciatica at short-term follow-up. Br J Gen Pract 2002;52: 119-23.

12. Alentado VJ, Mroz TE, Lubelski D, et al. Optimal duration of conservative management prior to surgery for cervical and lumbar radiculopathy: a literature review. Glob Spine J 2014;4: 279-86.

13. Lewis R, Williams N, Matar HE, et al. The clinical effectiveness and cost-effectiveness of management strategies for sciatica: systematic review and economic model. Health Technol Assess 2011;15:1-578.

14. Thomas KC, Fisher CG, Boyd M, et al. Outcome evaluation of surgical and nonsurgical management of lumbar disc protrusion causing radiculopathy. Spine 2007;32:1414-22.

15. Lau PM, Chow DH, Pope MH. Early physiotherapy intervention in an accident and emergency department reduces pain and improves satisfaction for patients with acute low back pain: a randomised trial. Aust J Physiother 2008;54:243-9.

16. Wand BM, Exspsc G, Bird C, et al. Early intervention for the management of acute low back pain education, manual therapy, and exercise. Spine 2004;29:2350-6.

17. Whitfill T, Haggard R, Bierner SM, et al. Early intervention options for acute low back pain patients: a randomized clinical trial with one-year follow-up outcomes. J Occup Rehabil 2010;20:256-63.

18. Fritz JM, Childs JD, Wainner RS, et al. Primary care referral of patients with low back pain to physical therapy: impact on future health care utilization and costs. Spine 2012;37:2114-21.

19. Gellhorn AC, Chan L, Martin B, et al. Management patterns in acute low back pain: the role of physical therapy. Spine 2012;37:775-82.

20. Teare MD, Dimairo M, Shephard N, et al. Sample size requirements to estimate key design parameters from external pilot randomised controlled trials: a simulation study. Trials 2014;15:264.

21. Ritchie J, Lewis J. Qualitative research practice- a guide for social science students and researchers. Sage, 2003.

22. Coppieters MW, Butler DS. Do "sliders" slide and "tensioners" tension? An analysis of neurodynamic techniques and considerations regarding their application. Man Ther 2008;13:213-21.

23. O'Sullivan P. Diagnosis and classification of chronic low back pain disorders: maladaptive movement and motor control impairments as underlying mechanism. Man Ther 2005;10:242-55.

24. Luomajoki H, Moseley GL. Tactile acuity and lumbopelvic motor control in patients with back pain and healthy controls. $\mathrm{Br} J$ Sports Med 2011;45:437-40

25. Hengeveld E. Maitlands' Vertebral Manipulation. 8th edn. Edinburgh: Churchill Livingstone, 2013:179-210.

26. Nicholas MK, George SZ. Psychologically informed interventions for low back pain: an update for physical therapists. Phys Ther 2011;91:765-76.

27. Linton SJ, Shaw WS. Impact of psychological factors in the experience of pain. Phys Ther 2011;91:700-11.

28. Blyth FM, Macfarlane GJ, Nicholas MK. The contribution of psychosocial factors to the development of chronic pain: the key to better outcomes for patients? Pain 2007;129:8-11.

29. Airaksinen O, Hildebrandt J, Ursin H, et al. Chapter 4. European guidelines for the management of chronic non-specific low back pain. Eur Spine J 2006;2006;15(suppl 2):S192-300.

30. Otoo SKW, Hendrick P, Ribeiro D. The comparative effectiveness of advice/education compared to active physiotherapy (manual therapy and exercise) in the management of chronic non-specific low back pain. Phys Ther Rev 2015;20:16-26.

31. Searle A, Spink M, Ho A, et al. Exercise interventions for the treatment of chronic low back pain: a systematic review and meta-analysis of randomised controlled trials. Clin Rehabil 2015;29:1155-67.

32. Pinto RZ, Maher CG, Ferreira ML, et al. Drugs for relief of pain in patients with sciatica: systematic review and meta-analysis. $B M J$ 2012;13:e497-e497.

33. Craig $\mathrm{P}$, Dieppe $\mathrm{P}$, Macintyre $\mathrm{S}$, et al. Developing and evaluating complex interventions: the new medical research council guidance. BMJ 2008;337:a1655.

34. Bellg AJ, Borrelli B, Resnick B, et al. Enhancing treatment fidelity in health behavior change studies: best practices and recommendations from the NIH behavior change consortium. Health Psychol 2004; 23:443-51

35. Moher D, Hopewell S, Schulz KF, et al. CONSORT 2010 explanation and elaboration: updated guidelines for reporting paralle group randomised trials. Int J Surg 2012;10:28-55.

36. Boeije H. A purposeful approach to the constant comparative method in the analysis of qualitative interviews. Qual Quant 2002;36:391-409.

37. Guest G, MacQueen KM, Namey EE. Introduction to applied thematic analysis. Applied Thematic Analysis. 2012:3-20.

38. Lincoln YS, Guba EG. Naturlistic enquiry. Sage, 1984

39. O'Cathain A, Murphy E, Nicholl J. Three techniques for integrating data in mixed methods studies. BMJ 2010;341:1147-50. 